\title{
Perceptual organization of auditory temporal patterns in European starlings (Sturnus vulgaris)
}

\author{
RICHARD F. BRAATEN and STEWART H. HULSE \\ The Johns Hopkins University, Baltimore, Maryland
}

\begin{abstract}
The perception of continuously repeating auditory patterns by European starlings was explored in seven experiments. In Experiment 1, 4 starlings learned to discriminate between two continuously repeating, eight-element, auditory patterns. Each eight-element pattern was constructed from different temporal organizations of two elements differing in timbre. In Experiments 2-7, the repeating patterns were transformed in ways designed to identify the starlings' perceptual organization of the patterns. In Experiment 2, the starlings identified patterns beginning with novel starting points. In Experiment 3, discrimination performance was adversely affected by reorganizing the elements in the patterns. In Experiments 4 and 5, the pattern elements were altered. In Experiment 4, the patterns were constructed from two novel elements. In Experiment 5, the temporal location of the two pattern elements was reversed. The transformations of the patterns in Experiments 4 and 5 affected discrimination performance for some, but not all, of the starlings. In Experiments 6 and 7, replacing either of the two elements with silent intervals had no effect on discrimination performance. The results of these experiments identify basic grouping principles that starlings use when they perceive auditory patterns.
\end{abstract}

When a songbird hears a series of acoustic elements, what does it perceive? Does it perceive a series of unrelated elements, or are the elements organized by perceptual rules? In this paper, we address this question by studying the discrimination and perceptual organization of auditory temporal patterns by European starlings.

It is known that human perception makes great use of perceptual grouping to organize auditory stimulation. This is apparent, for example, in the way in which frequency components are grouped in complex tones and in phonemic structures (Bregman, Abramson, Doehring, \& Darwin, 1985; Chalikia \& Bregman, 1989; Dannenbring \& Bregman, 1978; McAdams, 1989), or in which the consecutive notes in music are perceptually organized to form a melody (Attneave \& Olson, 1971; Deutsch, 1982; Dowling \& Harwood, 1986). The principles that govern the grouping of acoustic elements into meaningful structures has been a topic of a good deal of research in human auditory perception (Bregman, 1990). This research has pointed to the importance of perceptual grouping for constructing meaningful units of perception from acoustic elements.

This paper represents a portion of a dissertation presented to The Johns Hopkins University by R.F.B. in partial fulfillment of the $\mathrm{PhD}$ requirement. Research was supported by National Science Foundation Grant BNS-8911046 to S.H.H. We thank Dan Bernard, Cynthia Gray, Sue Page, and Annie Takeuchi for their comments on the research. We also thank Albert Bregman, Jay Dowling, and an anonymous reviewer for comments on an earlier version of the manuscript. The starlings were provided by Christian Grue of the Patuxent Federal Wildife Preserve, Laurel, MD. Correspondence should be addressed to R.F. Braaten, Department of Psychology, Center for Infant Development, University of Denver, Denver, CO 80208.
Recently, comparative psychologists have begun to study animals' perception of relationships among acoustic elements. A number of experiments have been conducted to study the perception of serial frequency relationships (Braaten, Hulse, \& Page, 1990; D'Amato, 1988; D'Amato \& Salmon, 1982, 1984; Dooling, Brown, Park, Okanoya, \& Soli, 1987; Hulse \& Cynx, 1985, 1986; Hulse, Cynx, \& Humpal, 1984; Hulse, Page, \& Braaten, 1990b; Page, Hulse, \& Cynx, 1989). Such studies have generally involved training animals to discriminate among two or more patterns. The patterns are then transposed, changing the frequencies of elements of the patterns, but maintaining the relational frequency structure among the pattern elements. In general, this body of research has shown that animals show a preference for solving perceptual problems on the basis of the absolute frequency of pattern elements. Under some conditions, however, especially when absolute frequency cues are mitigated, perception of relational frequency structure emerges as a secondary strategy (see Hulse, Page, \& Braaten, 1990a, for a review of this work).

In the experiments on the perception of frequency structure, animals have been presented with acoustic patterns with a very definite formal frequency structure. For example, many experiments require songbirds to discriminate sequences in which successive elements increase monotonically in frequency from sequences in which the elements decrease monotonically in frequency. The songbirds are then presented with transpositions of the sequences, to determine whether the animals have learned the formal structure that was available to them.

In the experiments reported here, we used a new approach to the study of perceptual organization in starlings. 
This approach was adapted from Garner's studies of human auditory perceptual organization (Garner, 1974; Royer \& Garner, 1966, 1970). Rather than present starlings with auditory patterns with a definite formal structure, we presented the birds with sequences in which the formal structure was ambiguous. Transfer tests were then designed to determine whether the starlings selected a perceptual organization from among the many possible organizations of the ambiguous sequences. Before turning to these experiments with starlings, we first review some of the work on human perception of ambiguous auditory patterns.

\section{Human Perception of Continuously Repeating Auditory Patterns}

Many experiments on human auditory perceptual organization have made use of continuously repeating patterns (e.g., Garner \& Gottwald, 1968; Handel, 1974; Handel \& Lewis, 1970; Handel \& Todd, 1981; Preusser, 1972; Preusser, Garner, \& Gottwald, 1970; Royer \& Garner, 1966, 1970; Warren, Obusek, Farmer, \& Warren, 1969). Subjects are asked to listen to and identify a pattern. For example, consider a continuously repeating, dichotomous auditory pattern such as XXXXOOOOXXXXOOOO ..., where $X$ and $O$ represent two distinct elements, such as two tones of different timbre and pitch. When such a pattern is repeated for a sufficient duration, people eventually perceive a repeating pattern (Royer \& Garner, 1966, 1970). A number of organizations of the stimulus pattern are possible, but people perceive only a few of them. In the given example, people will usually perceive the repeating pattern to be XXXXOOOO, rather than another possible organization, XOOOOXXX. In the perception of these patterns, there is structure in the stimulus. Thus, in the example, nobody perceives the pattern to be XOXOXOXO. But the structure is also in the perceiver. People are selective about the structures they will perceive. Although perception is not imposed on a structureless world, perception is selected from a number of alternatives. Such experiments have shown that a few simple rules operate in the determination of perceptual organization. Among the rules that operate on human perceptual organization, those based on grouping by similarity are most relevant here.

\section{Grouping by Similarity}

Several experiments have shown that similarity is a powerful grouping principle in the perception of auditory patterns (Garner \& Gottwald, 1968; Handel \& Buffardi, 1968; Royer, 1967; Royer \& Garner, 1966, 1970). Thus subjects have listened to and identified repeating patterns, verbally, in writing (as a series of Xs and Os), or with a motor response (tapping out the pattern). For example, the subject might hear the pattern OOXXOOOOOOXXOOOOOOXXOOOO .... The subject would listen to the pattern until he/she could identify it. For any $n$-element repeating pattern, there are $n$ possible correct responses. In the example, most people $(54 \%)$ reported hearing OOOOOOXX, but a large minority (30\%) reported hear- ing XXOOOOOO (Royer \& Garner, 1966). Identifications of these repeating patterns show that similar elements are grouped together as much as possible. Only rarely does the perceived pattern break apart similar elements. In the example pattern, only $.08 \%$ of the subjects perceived the repeating pattern as XOOOOOOX. Furthermore, subjects tended to perceptually organize the pattern independently of its starting point. Identification of the patterns was faster when they began at the subjects' preferred starting points (e.g., OOOOOOXX in the example), and subjects made fewer errors in such instances (Royer \& Garner, 1966). This shows that perception is an active process in which the series of elements is reorganized to match the subjects' preferred perception, as determined by runs of similar elements.

\section{Auditory Streaming}

A common effect of grouping of acoustic elements by similarity is that one line of the stimulus is perceived as a figure against the background of the rest of the stimulus (Bregman \& Campbell, 1971; Divenyi \& Hirsh, 1978; Preusser et al., 1970). This phenomenon has been termed auditory streaming (Bregman \& Campbell, 1971). For example, if $X$ is a $2000-\mathrm{Hz}$ tone and $\mathrm{O}$ is a $400-\mathrm{Hz}$ tone, then, at certain rates, the continuously repeating pattern XOXOXXOO ... will split apart into two perceptual streams, $\mathrm{X}-\mathrm{X}-\mathrm{XX}-\mathrm{-} . .$. and $-\mathrm{O}-\mathrm{O}-\mathrm{-OO} \ldots$ Attention can be shifted to the elements of either of the two frequency streams, which becomes the figure, but it is extremely difficult to attend to both streams at once. The functional importance of auditory streaming is that it allows the organism to attend selectively to relevant information from one sequence while ignoring irrelevant background noise.

\section{The Present Experiments}

The series of experiments reported in this paper was designed to uncover rules of perceptual organization that operate in starlings' perception of auditory patterns. In all the experiments, starlings were required to discriminate between two eight-element, dichotomous, continuously repeating patterns, similar to those used by Royer and Garner (1966, 1970).

In Experiment 1, we describe acquisition of the baseline discrimination. During acquisition, the patterns were presented with seven different starting points in an attempt to force the bird to rely on the organization between the elements rather than on any individual pattern element. The patterns were repeated many times (16), to allow the bird the opportunity to perceive the temporal structure of the pattern. Each subsequent experiment represents a transfer from the baseline discrimination to patterns that were transformed in some way.

Each transfer test was designed to yield results that converged on the issue of temporal pattern organization. In Experiment 2, the starlings were presented with 2 starting points that had not been presented during baseline training, along with the 14 baseline starting points. In Experiment 3 , we were concerned with the starlings' per- 
ception of the overall structure of the patterns. In this experiment, the pattern elements remained unchanged, but the organization of the elements was altered. In Experiments 4 and 5, we examined the extent to which the discrimination depended on the particular elements that made up the patterns. In these experiments, the elements of the patterns were changed, but the organization of the elements remained constant. In Experiments 6 and 7, we addressed the possibility that the starlings perceived the sequences as perceptual streams; here, we were concerned with the starlings' discrimination of the patterns when either of the elements was replaced with a silent interval.

\section{EXPERIMENT 1 Discrimination of the Baseline Temporal Patterns}

The starlings' acquisition of a discrimination between two repeating temporal patterns is reported here. The patterns were composed of the same two elements in different temporal organizations. These elements were tones with the same fundamental frequency, but differing in timbre. Each pattern was eight elements in length and was repeated 16 times. The patterns could begin at any of seven starting points. The variable starting point was designed to encourage perception of the organization of the elements. Because it is possible that the birds could perceive different starting points of the same patterns as being different patterns, the eighth starting point for each pattern was not presented initially. This pattern was reerved for later testing (see Experiment 2).

\section{Method}

Subjects. Four male European starlings served as subjects (G24, G27, S53, and P64). Starling G24 was experimentally naive. Starlings G27 and S53 had previous experience in pilot work in discriminating two repeating patterns other than those in these experiments. Starling P64 had prior experience in a song preference experiment.

The starlings in these experiments were caught wild. They were obtained from the Federal Wildlife Preserve in Laurel, MD. The birds were food deprived to approximately $85 \%$ of their ad-lib weights. They received a diet of Purina Meat Builder (Purina, St. Louis, MO), chopped dog chow, carrots, and hard-boiled eggs (including shells) in the test chambers. They were supplemented with additional feedings of Purina Meat Builder in their home cages if necessary, to maintain their deprived weight. Grit was available in the home cages, and water was available at all times. The birds were housed in an aviary that contained approximately 50-60 starlings. Fluorescent full-spectrum lights were controlled by a timer that maintained the light:dark cycle consistent with that in Baltimore.

Apparatus. Acoustic stimulus generation, control of experimental contingencies, and data recording were controlled by an AT-type personal computer. The stimuli were generated by computer programs and stored digitally on disk. A DT2801 Data Translation (Marlborough, MA) D/A converter with a sampling rate of $20 \mathrm{kHz}$ fed the signal to a $10-\mathrm{kHz}$ low-pass filter, and then to a Coulborn (Lehigh Valley, PA) S84-04 rise/fall gate set at a linear rise/fall time of 6 msec. Output from the gate was sent to a Crown (Elkhart, IN) D-75 amplifier, and then to a Jensen (Lincolnshire, IL) 14-cm coaxial speaker mounted in the ceiling of an Industrial Acoustics (Bronx, NY) Model AC3 acoustic chamber.
A standard operant response panel, with internal measurements of $60 \times 80 \times 60 \mathrm{~cm}$ was mounted inside the acoustic chamber. A three-sided welded wire test cage measuring $28 \times 30 \times 20 \mathrm{~cm}$ was attached to the response panel, which formed the fourth side of the bird's test quarters. The response panel consisted of a row of three translucent response keys, $2 \mathrm{~cm}$ in diameter, and spaced $6 \mathrm{~cm}$ apart (center to center). Pecks on the keys were detected by microswitches. A food hopper delivered reward to a $6.0 \times 4.5 \mathrm{~cm}$ opening located $4.5 \mathrm{~cm}$ below the row of keys. Some birds received rewards from two hoppers, one located below each of the side keys; other birds received rewards from a single central hopper located below the center key. Indirect illumination (houselight) was provided by a translucent light source mounted on the side of the acoustic chamber. The chamber speaker was located $22 \mathrm{~cm}$ above the test cage.

Stimuli. Both patterns were constructed from the same two elements, identified here as $X$ and $O$. These elements had unique spectral structures, or timbres. Element $X$ contained equal energy at two harmonics - the fundamental frequency and the fourth harmonic. Element $O$ contained equal energy at three harmonics-the fundamental frequency, second harmonic, and third harmonic. Spectral structure is an important component of timbre, and starlings easily discriminate between these two timbres (Braaten \& Hulse, 1991).

The elements were created by computer software that summed sine waves of equal intensity. Both elements were presented with a fundamental frequency of $1000 \mathrm{~Hz}$. Therefore, Element $\mathrm{X}$ contained equal energy at 1000 and $4000 \mathrm{~Hz}$. Element $O$ contained equal energy at 1000,2000 , and $3000 \mathrm{~Hz}$. The two elements were equated for overall intensity, and sine-wave components of each element were produced in phase.

The elements were presented with a duration of $100 \mathrm{msec}$. Each tone was passed through a rise/fall gate with a linear onset/offset time of $6 \mathrm{msec}$. The elements were presented at an intensity level that ranged, between sessions, from 72 to $77 \mathrm{~dB}(\mathrm{~A})$. The sound level was recalibrated periodically to $75 \mathrm{~dB}(\mathrm{~A})$, using a Rion (Tokyo, Japan) NA-20 sound-level meter.

From these two elements, two patterns were constructed. In Pattern A, the elements were organized as XXXXXOOO. In Pattern B, they were organized as OXOXOOXX. The 100 -msec elements were separated by 100 -msec interelement intervals, so the patterns were presented at a rate of 5 elements/sec. The patterns repeated continuously, with no pause between repetitions, for up to $25.6 \mathrm{sec}$, or 16 repetitions. On any given trial, the patterns could begin at seven of the eight possible starting points for each pattern. For example, two versions of Pattern B, with different starting points, were OXOXOOXX and XOXOOXXO. Versions XXXOOOXX for Pattern A and OXOOXXOX for Pattern B were not presented initially. These versions were reserved for future testing in Experiment 2.

Baseline discrimination. The birds discriminated between the two patterns in a two-alternative, forced choice procedure. The starlings were required to withhold responses for a 25.6-sec listening period. Responses to the side keys during the listening period turned off the sound and extinguished the houselight for a time-out period of $10 \mathrm{sec}$. The offset of sound presentation at the end of the 25.6-sec listening period served as the signal for the bird to respond to one of the side keys.

It was necessary to train the birds, through the use of a gradual shaping procedure, to wait until the end of the listening period before responding. In the shaping procedure, the starlings were presented with one of two continuously repeating tones on each trial. These tones were 880 - and $1480-\mathrm{Hz}$ sine waves. The birds were rewarded for responding to the left key in the presence of the 880$\mathrm{Hz}$ tone, and to the right key for the $1480-\mathrm{Hz}$ tone. Responses to the incorrect key were punished with a 10-sec time-out period. The listening period was initially $1.6 \mathrm{sec}$ during the shaping procedure. Responses prior to the end of this listening period resulted in the 10-sec time-out. As the birds learned to withhold responses, the listening period was extended. Eventually, the listening period was 
made variable, so that the birds could not simply time a fixed period before responding. The exact listening periods that were used varied between birds, but all the starlings eventually learned to wait for a mean duration of $25 \mathrm{sec}$ before responding. The shaping period continued for a period of about 5 weeks, at which point the birds were waiting reliably until the end of the listening period before making a response. At this point, training of the pattern discriminations began.

During discrimination training, each trial began with the center keylight illuminated. A peck to the center key darkened that key and initiated presentation of one of the two patterns. The patterns were selected randomly $(p=.5)$ for each trial, with the constraint that the same pattern was not presented on more than four consecutive trials. Within each pattern, one of the seven starting points was also selected randomly $(p=.14)$.

Pattern presentation was followed by a 10 -sec response period. During the response period, responses to the left key following presentation of Pattern A resulted in $3 \mathrm{sec}$ of access to the raised, left food hopper. Responses to the right key following presentation of Pattern B were rewarded with $3 \mathrm{sec}$ of access to the right hopper. Incorrect responses to either key were punished with a 10-sec timeout period. A failure to respond during the response period also resulted in the time-out period. A 5-sec intertrial interval, with the houselight on, followed either food reward or time-out.

To avoid key biases, correction trials were used throughout the experiment. When the bird made an incorrect response, it received the same pattern on the following trial. This procedure was instituted so that the starling could not simply respond to the same key on each trial, which would result in food reward on $50 \%$ of the trials. Correction trials followed incorrect responses of any type, including a response to the wrong key, a response during the listening period, or a failure to respond during the response period. Data from correction trials are not included in any of the analyses.

The starlings worked in daily, 1.5- to 2-h sessions, during which time they received approximately 50-175 trials.

Transfer tests. The specific transfer tests are described in Experiments 2-7. However, some general features of the transfer tests can be described here.

Transfers began once the birds were discriminating at a performance level of at least $67 \%$ correct, averaged over five sessions. All birds received at least 160 trials for each transfer test (not counting correction trials). The transfer tests followed at least 160 trials of baseline discrimination. Following the transfer, the birds were returned to at least 160 trials of baseline. This procedure continued until all the transfer tests were completed. Transfer tests began the day following the one on which the bird had reached at least 160 baseline trials. For most transfer tests, this meant that the birds received two to three daily baseline sessions, followed by two to three daily transfer sessions. The first 160 trials of transfer for each bird were analyzed in eight blocks of 20 trials.

Baseline performance was not always stable, both because of the difficult nature of the task, and because transfer tests sometimes disrupted baseline performance. Therefore, a baseline criterion of at least $60 \%$ correct was instituted. If the first 160 trials following a transfer were not at least $60 \%$ correct, the bird continued the baseline discrimination until it was at least $60 \%$ correct for two consecutive sessions. Once this criterion was regained, the following 160 trials were counted as the baseline performance against which the subsequent transfer performance was measured.

During all transfer tests, reinforcement contingencies remained in effect.

Here, the experiments will be described in a conceptual order, not the order in which they were actually done. The chronological order of transfers was the same for all birds: (1) novel starting points (Experiment 2); (2) novel elements (Experiment 4); (3) reversed elements (Experiment 5); (4) X element only (Experiment 6); (5) O element only (Experiment 7); (6) reorganized elements (Experiment 3).

\section{Results}

All 4 starlings learned to discriminate between the two baseline patterns. Figure 1 shows the acquisition curves for the 4 birds, from the initial session until the time of the first transfer. Each data point in Figure 1 represents the average of five consecutive sessions (the last data point for G24 and P64 represents an average of four sessions). The discrimination was difficult for 2 of the starlings. The other 2 starlings, however, learned the discrimination relatively quickly. G27 learned the task the fastest, reaching a discrimination level of $67 \%$ correct after 30 sessions. G24 was discriminating correctly on $74 \%$ of the trials after 39 sessions. Two of the birds, S53 and P64, took much longer to learn the task. S53 reached a performance level of $69 \%$ correct after 165 sessions; P64 reached $67 \%$ correct after 164 sessions. The mean performance of $69.2 \%$ at the time of the initial transfer was significantly greater than chance $(50 \%)[t(3)=11.65, p<.01]$.

\section{Discussion}

All 4 starlings were able to discriminate between the repeating patterns. This was true even though the continuous repetition of the patterns, the use of the same two elements in each of the patterns, and the variable starting points made it unlikely that the starlings could have been discriminating on the basis of some local feature of the patterns. The experiment was designed to encourage the starlings to attend to the global relations among the pattern elements. The difficulty that Starlings S53 and P64 had in learning the discrimination may have resulted from a difficulty in perceiving the relations among pattern elements. Starlings very quickly learn discriminations between single tones (Braaten, 1991) and will solve pitch pattern discriminations on the basis of the absolute frequency of individual tones when given the opportunity, rather than on the basis of the relationships among tones (Hulse \& Cynx, 1986; Hulse et al., 1984). In fact, in an earlier experiment that required attention to global pitch relationships, starlings were unable to learn a discrimi-

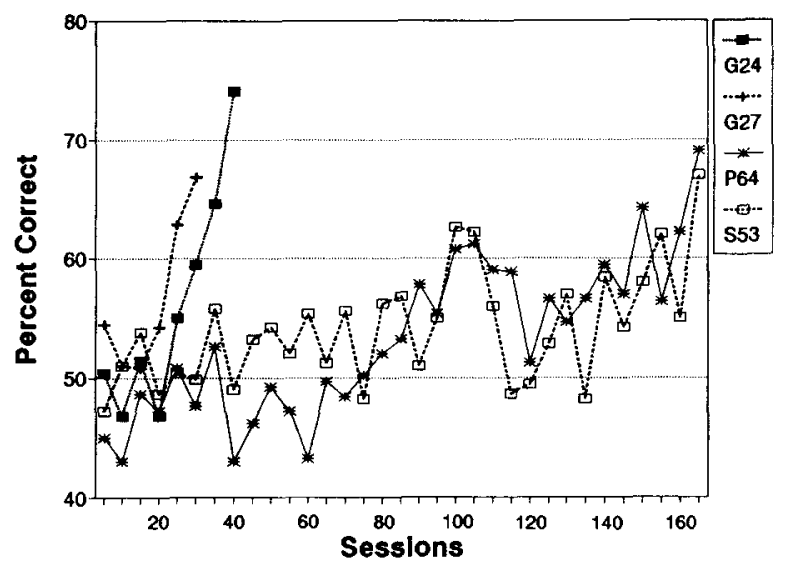

Figure 1. Acquisition of the baseline discrimination by the 4 starlings in Experiment 1. Each data point represents the mean discrimination performance of five daily sessions. 
nation between rising and falling pitch patterns (Page et al., 1989).

There were very large individual differences in acquisition. The discrimination was learned quickly by 2 of the starlings, and slowly by the 2 others. The source of these differences is not apparent. G27 and S53 had prior experience in discriminating other repeating patterns; yet G27 acquired this discrimination relatively rapidly, whereas S53 was very slow. Starling G24, which had no prior laboratory experience, also acquired the discrimination more rapidly than the 2 slower birds.

It is important to emphasize that the reason for the relatively high percentage of errors in discrimination of the repeating patterns is not necessarily due to perceptual deficits in the starling. The nature of the task could very plausibly have created other difficulties. For example, the starlings may have had trouble remembering the correct response from the time at which a response decision was made until the end of the listening period. The starlings were quite good at waiting the required $25.6 \mathrm{sec}$ from stimulus onset until response time. Over the last three sessions of training prior to the transfer tests, the starlings responded during the listening period on only $2 \%$ of the trials. Although the purpose of the lengthy listening period was to give the birds plenty of time to perceive the structure of the pattern, it is possible that a shorter listening period would have resulted in higher performance.

\section{EXPERIMENT 2 Novel Starting Points}

The starlings may have learned the discrimination on the basis of the repeating organization of the pattern elements. Alternatively, the starlings may have been learning serial lists of elements. That is, the starlings may not have perceived the periodicity of the repeating patterns. Rather, the 14 starting points may have been perceived as 14 separate sequential lists of elements. This seems unlikely, because it would have required the starlings to learn 14 separate lists. However, animals have been known to classify large numbers of visual stimuli by rote memory when much simpler classification schemes seem apparent to human observers (Greene, 1984).

In this experiment, the starlings were presented with the 2 novel starting points, along with the 14 baseline starting points, of the baseline patterns. It was expected that if the starlings perceived the periodicity of the pattern organization, performance with the novel starting points would be no different than performance with the baseline starting points. If, on the other hand, the starlings had learned 14 separate lists of elements, performance on the novel starting points would be much worse than baseline.

\section{Method}

Subjects. The 4 starlings that acquired the baseline discrimination served as subjects.
Apparatus. The apparatus was described in Experiment 1. Stimuli. In this transfer, the birds received the two starting points for each of the two patterns that had not been received during baseline discrimination. These two patterns were the XXXOOOXX version of Pattern A, and the OXOOXXOX version of Pattern B.

Procedure. The novel starting points were presented in transfer sessions along with the 14 starting points that the birds had learned. Therefore, on each trial, the probability of receiving each pattern remained .5 , but the probability of receiving any particular starting point, given a particular pattern, was .125 instead of .14. The starlings continued with transfer sessions until they had received 20 trials with each of the 16 starting points (for a total of 320 trials).

\section{Results}

Performance was no different with the novel starting points than it was with the baseline starting points. Figure 2 shows means for the 4 birds on the initial sessions that included the two novel starting points. The mean performance for the first 20 trials of each of the 14 baseline starting points and the mean performance of the first 20 trials of the 2 novel starting points are shown for each starling. For the 4 starlings combined, the mean performance of $70 \%$ correct out of the 20 trials was not significantly better than the $69 \%$ correct that the birds achieved with the novel starting points $[t(3)=0.54, p>.05]$.

\section{Discussion}

Performance with the novel starting points provides evidence that the starlings perceived the organization among the elements of the baseline patterns. These patterns were identified as accurately as were the patterns with the 14 familiar starting points. Apparently, the starlings did not perceive the patterns that started at different points as being different. Rather, they perceived some constant property of the patterns that emerged through the repetition

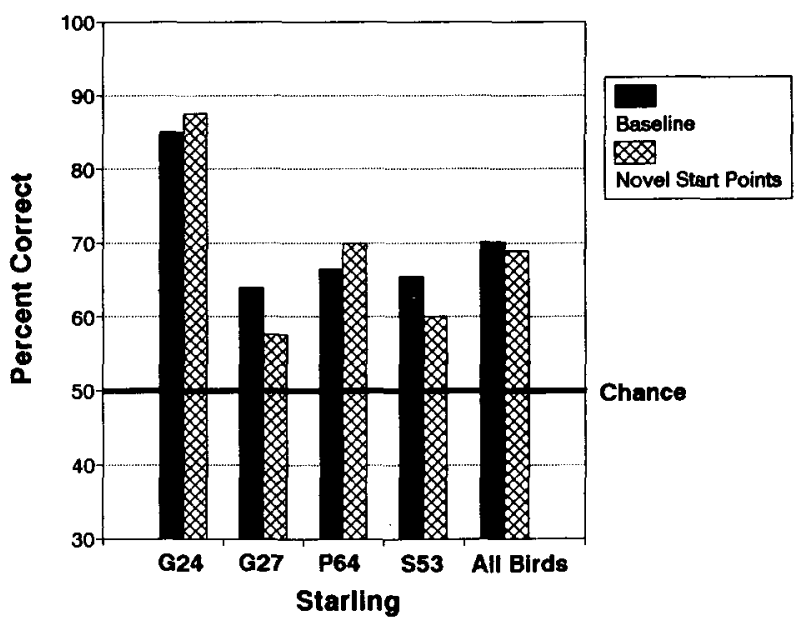

Figure 2. Baseline and transfer discrimination performances for the starlings in Experiment 2. Baseline data are the mean performances on the first 20 trials of the 14 baseline starting points presented during transfer sessions. Transfer data are the mean performances on the first 20 trials of the $\mathbf{2}$ novel starting points presented during transfer sessions. 
of the patterns. The exact nature of this constant property was investigated in subsequent experiments.

\section{EXPERIMENT 3 Reorganization of Elements}

This experiment was designed to explore the nature of the starlings' discrimination. One possibility is that the starlings were attending to the relative proportion of the elements in each of the patterns, and not to the pattern organization. In this experiment, that proportion was maintained, but the organization of the elements within the pattern was changed. In the baseline patterns, Pattern A contained five Xs and three Os, and Pattern B contained four Xs and four Os. It was expected that if the starlings were attending simply to the relative proportion of the elements in each of the patterns, this transfer would not cause a change in discrimination performance, because that feature would be maintained. If, however, the starlings were attending to the organization of the elements within the patterns, performance would be disrupted substantially.

\section{Method}

Subjects. The same 4 starlings served as subjects.

Apparatus. The apparatus is described in Experiment 1.

Stimuli. The same two elements were used in this transfer. The $X$ and $O$ elements were presented in the same proportion as baseline for each pattern (five Xs for Pattern A, four Xs for Pattern B). The elements were reorganized to form two new patterns. Pattern $A$ became XXOXOXXO, and Pattern B became XXXXOOOO. The new patterns could begin at any of the eight possible starting points.

Procedure. Each bird received at least 160 trials with the new patterns, as described for the transfer tests in Experiment 1.

\section{Results}

The starlings were unable to identify the transfer patterns with the reorganized elements. The discrimination of $46.9 \%$ on the transfer patterns was much worse than the baseline discrimination of $71.3 \%[t(7)=13.34, p<$ $.01]$. Figure 3 shows that this drop in performance was consistent. Three of the birds, G24, G27, and P64, showed a significant drop in discrimination $[t \mathrm{~s}(7)=$ $10.68,3.82$, and 3.75 , all $p s<.01$ ]. S53's drop from $65.6 \%$ to $54.4 \%$ was not significant $[t(7)=2.11, p>$ $.05]$, but neither was the transfer performance better than chance $[t(7)=1.51, p>.05]$.

None of the birds identified the two transfer patterns at a level higher than chance. Starlings G27, P64, and S53 were no different from chance $[t \mathrm{~s}(7)=-0.55$, -0.57 , and 1.51 , respectively, all $p s>.05]$. Starling G24's performance of $36.9 \%$ was actually worse than chance $[t(7)=-2.54, p<.05]$.

\section{Discussion}

The results of this transfer provide strong evidence that the starlings perceived the organization among the elements in the patterns. Reorganizing the elements of the two patterns seriously disrupted the ability of the starlings

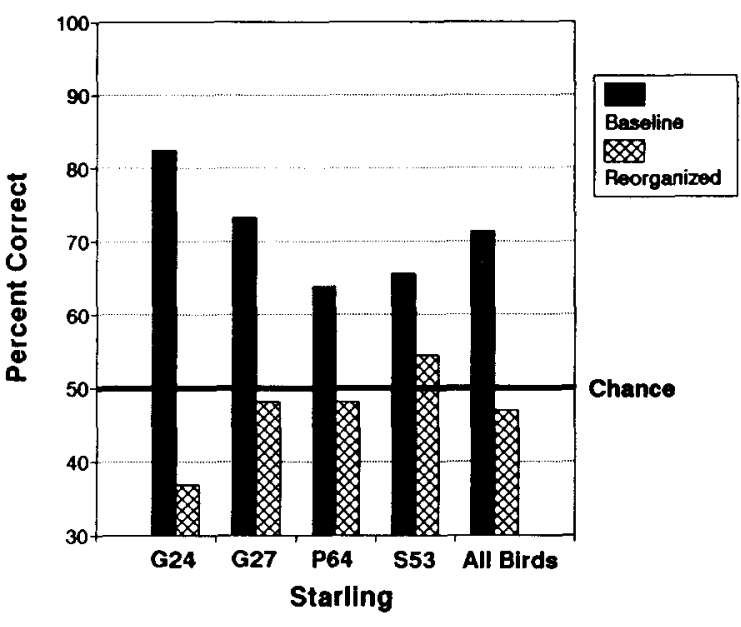

Figure 3. Baseline and transfer discrimination performances for the starlings in Experiment 3. Baseline data are the mean baseline performances on the eight blocks of 20 trials preceding the transfer test. Transfer data are the mean transfer performances on the eight blocks of $\mathbf{2 0}$ trials with the stimulus elements reorganized.

to identify the patterns. This was true for all the birds. Simply perceiving the relative proportion of the elements, which differed between the two patterns, could not account for the poor performance in this transfer test.

It is interesting that Starling G24's performance suffered the most on this transfer test. This starling consistently had the highest baseline level of performance, yet, with the reorganized patterns, this starling was the worst of the 4 birds at identifying the patterns. This suggests that the reason for G24's rapid acquisition and high level of baseline discrimination is that it, more than the other starlings, learned to attend to the organization of the pattern elements (this suggestion is also supported by the results of Experiments 4 and 5).

G24's performance also gives a clue to how the patterns were organized perceptually, at least for that 1 starling. In the baseline patterns, Pattern A consisted of two runs of identical elements, XXXXXOOO. Pattern B had six runs of identical elements, OXOXOOXX. With the transfer patterns, Pattern A had six runs of identical elements, XXOXOXXO, and Pattern B had two runs of identical elements, XXXXOOOO. In the transfer test, G24 treated Pattern A as though it was the baseline Pattern B, and Pattern B as though it was the baseline Pattern A. This reversal of performance strongly suggests that G24 was grouping identical elements perceptually. Humans also group identical elements in patterns such as these; descriptions of the repeating patterns rarely break up groupings of identical elements (Royer \& Garner, 1966, 1970).

\section{EXPERIMENT 4 Novel Elements}

The starlings were able to learn the baseline discrimination despite the variable starting point. Furthermore, 
they were able to identify patterns with novel starting points and were not able to identify patterns in which the elements were reorganized. This suggests that the discrimination was not based on features of individual pattern elements, but on the organization of the elements in the patterns. In Experiment 4, we tested more directly the idea that the discrimination was independent of individual pattern elements. In this transfer test, the two pattern elements were replaced with novel elements. To the extent that the baseline discrimination was independent of the elements, it was expected that the starlings would maintain the discrimination with the novel elements.

\section{Method}

Subjects. The 4 starlings from Experiment 1 served as subjects. Apparatus. The apparatus is described in Experiment 1 .

Stimuli. The frequency and timbre of the elements were changed. The $\mathrm{X}$ element was changed to a sine wave with a fundamental frequency of $1200 \mathrm{~Hz}$. The $\mathrm{O}$ element was changed to a complex tone, also with a fundamental frequency of $1200 \mathrm{~Hz}$. The sine wave contained energy at the fundamental frequency only. The complex tone contained energy at the fundamental frequency and at the third and fifth harmonics. The amount of energy in the respective harmonics of the complex tone decreased with increasing frequency. The third harmonic was $6 \mathrm{~dB}$ less intense than the fundamental frequency, and the fifth harmonic was $11 \mathrm{~dB}$ less intense than the fundamental frequency. Intensity and duration of the elements were unchanged.

The new elements were created with the Digidesign (Menlo Park, CA) Softsynth additive synthesis software program on a Macintosh IIx computer.

Procedure. The starlings were transferred to the patterns with the novel elements for at least 160 trials, as described in the procedure for the transfer tests in Experiment 1. The temporal organization of the elements in the patterns remained unchanged.

\section{Results}

The 160 transfer trials were analyzed as eight blocks of 20 trials and compared with the eight blocks of 20 baseline trials that immediately preceded the transfer. These data are represented in Figure 4. The starlings were able to discriminate the patterns with novel elements. The average of $61.4 \%$ for the 4 birds was significantly above chance $[t(7)=7.18, p<.01]$. Performance was not as good as that with the baseline patterns, however. Discrimination dropped from an average of $70.9 \%$ correct for the 4 birds on the baseline trials to an average of $61.4 \%$ correct on the trials with novel elements $[t(7)=3.55$, $p<.01]$.

Figure 4 shows that the results are not consistent for the 4 birds. The performances of G24 and G27 were not significantly reduced by the novel elements $[t \mathrm{~s}(7)=1.17$ and 0.00 , respectively, $p s>.05]$. However, the novel elements clearly disrupted the discrimination of P64 $[t(3)=5.34, p<.01]$. Although the drop in performance for S53 was not statistically significant $[t(7)=$ $1.97, p>.05]$, neither was the performance with the novel elements different from chance for that bird $[t(7)=$ $2.24, p>.05]$.

\section{Discussion}

Two of the starlings showed that their discrimination of these patterns was independent of the elements that made

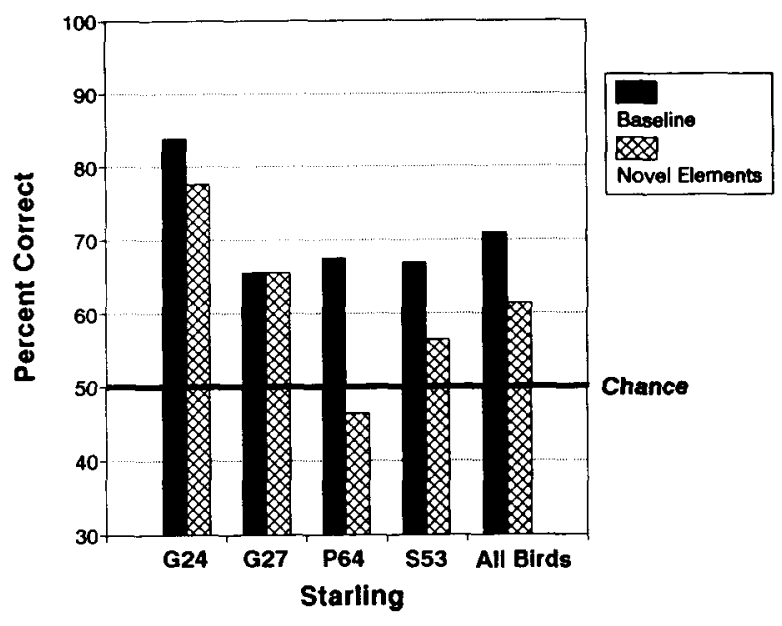

Figure 4. Baseline and transfer discrimination performances for the starlings in Experiment 4. Baseline data are the mean baseline performances on the eight blocks of 20 trials preceding the transfer test. Transfer data are the mean transfer performances on the eight blocks of 20 trials with the novel stimulus elements.

up the patterns. The present results, along with those of Experiments $1-3$, strongly suggest that these starlings perceived the organization of the elements in the patterns. The other 2 starlings' discrimination did depend on the particular elements used in baseline training. The 2 starlings that were unaffected by the transfer, G24 and G27, were those that acquired the discrimination relatively rapidly in Experiment 1. Perhaps the rapid acquisition was a result of these birds' attending to the overall structure of the pattern; attention to the elements may have interfered with acquisition of the task for Starlings P64 and S53.

\section{EXPERIMENT 5 Reversed Elements}

Experiment 4 showed that novel elements did not disrupt performance for 2 of the starlings. Experiment 5 tested the limits of the independence of perfomance from the pattern elements. In this transfer test, the pattern elements were reversed. The $X$ elements were replaced with the O elements, and the Os were replaced with the Xs. As in Experiment 4, this transfer maintained the overall temporal structure of the patterns.

Reversing the pattern elements was a very strong test of the starlings' perception of the relational organization of the elements. If the starlings perceived the relation of the elements to each other, independently of the elements themselves, reversing the elements would not result in a decrement in performance.

\section{Method}

Subjects. The same 4 starlings served as subjects.

Apparatus. The apparatus is described in Experiment 1.

Stimuli. The same two elements were used in this experiment. However, the organization of the elements was reversed. The locations of $\mathrm{X}$ elements in the pattern were replaced by the $\mathrm{O}$ element, and the locations of the $O$ elements were replaced by the $X$ 
element. Thus, the baseline Pattern A, XXXXXOOO, became OOOOOXXX, and the baseline Pattern B, OXOXOOXX, became XOXOXXOO.

Procedure. Each bird was transferred to the patterns with the reversed elements for at least 160 trials, as described in the procedure for the transfer test in Experiment 1.

\section{Results}

Reversing the pattern elements was more disruptive to the starlings' discrimination performance than were the novel elements. The performance of the starlings on the baseline patterns and the transfer is shown in Figure 5 . The $56.4 \%$ performance for the 4 birds on the transfer was significantly better than chance $[t(7)=4.64, p<$ $.01]$; however, this was entirely due to the $71.2 \%$ performance of G24 $[t(7)=7.20, p<.01]$. None of the other 3 starlings was above chance on the transfer patterns $[t s(7)=1.39,-1.59$, and 0.94 for $\mathrm{G} 27, \mathrm{P} 64$, and S53, respectively, all $p s>.05$ ].

The discrimination of $67.0 \%$ on the baseline patterns was significantly better than the $56.4 \%$ correct on the transfer patterns $[t(7)=5.15, p<.01]$. This drop in performance was consistent for the individual starlings. G24 and P64's performance was worse with the transfer patterns $[t(7)=6.42$ for $\mathrm{G} 24, p<.01$, and $t(7)=3.20$ for P64, $p<.05]$. Starling S53 was not significantly worse with the transfer patterns $[t(7)=0.94, p>.05]$, but neither was discrimination above chance with these patterns $[t(7)=0.94, p>.05]$. Starling G27's data could not be interpreted, because discrimination was not better than chance with the baseline patterns $[t(7)=1.42$, $p>$.05]

\section{Discussion}

Reversing the pattern elements was an even stronger test of the idea that the starlings were attending to some

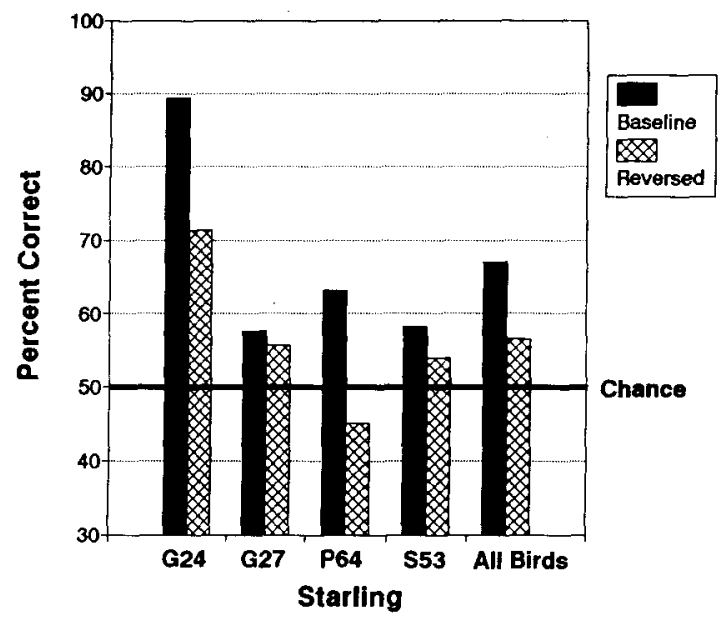

Figure 5. Baseline and transfer discrimination performances for the starlings in Experiment 5. Baseline data are the mean baseline performances on the eight blocks of 20 trials preceding the transfer test. Transfer data are the mean transfer performances on the eight blocks of 20 trials with the reversed stimulus elements. local feature of the patterns than were the novel elements of Experiment 4. Once again, Starling G24 showed strong evidence of perceiving organization among the elements. That starling was still able to discriminate between the patterns even when the elements were reversed. For 2 of the birds, S53 and P64, reversing the elements adversely affected discrimination performance. Unfortunately, G27's baseline performance was not above chance, so it is not known how the element reversal affected its discrimination.

Although altering the elements did affect discrimination performance of some of the starlings, it does not follow from the results of Experiments 4 and 5 that the starlings did not perceive the organizational structure of the elements. Starlings G24 and G27 still were able to discriminate the patterns with novel elements in Experiment 4, as was Starling G24 in Experiment 5. Furthermore, it is possible that the other 2 starlings also attended to the pattern organization as well as the pattern elements. Reversing the pattern elements also reversed the temporal organization associated with each of the elements. If the organization of one of the elements was perceived as a figure against the background of the other elements-that is, if the elements were perceived as two separate auditory streams-then reversing the elements might be expected to affect performance. This idea was explored further in Experiments 6 and 7.

\section{EXPERIMENT 6 Auditory Streaming: $\mathrm{X}$ Element as Figure}

Continuously repeating auditory patterns are sometimes segregated perceptually into separate streams (Bregman, 1990) by human listeners. This happens when attention is focused on one of the elements. The element that is attended to becomes the figure, and the other element becomes the background. The perceptual organization consists of the relationships between the figural element and the interelement intervals. The pattern is perceived as a continuously repeating rhythm.

To find out whether the starlings perceived the patterns as rhythmic streams, all instances of one of the elements were removed from both patterns and were replaced by silent intervals. If the starlings had been perceiving the patterns as rhythmic streams, this alteration of the patterns would not affect identification. On the other hand, if the starlings were attending to the relationships between the successive pattern elements, this transfer would disrupt performance.

In this experiment, all of the 100 -msec $\mathrm{O}$ elements were removed from the patterns and were replaced with 100 msec silent intervals.

\section{Method}

Subjects. The same 4 starlings served as subjects.

Apparatus. The apparatus is described in Experiment 1.

Stimuli. The $\mathrm{X}$ element remained the same as in the baseline patterns. The $\mathrm{O}$ element was replaced with a 100 -msec silent interval. 
Thus, Pattern A in this transfer was XXXXX---, where the dash represents a silent interval, and Pattern $B$ was $-X-X--X X$.

Procedure. Each starling received 160 trials of the transfer patterns, as described in the procedure for the transfer tests in Experiment 1 .

\section{Results}

Baseline and transfer performance for the 4 starlings are shown in Figure 6. The difference in performance between the baseline and the transfer patterns was not statistically significant $[t(7)=2.18, p>.05]$. This result was consistent for the 4 birds; removing the $O$ elements from the patterns did not significantly disrupt performance for any of the starlings $[t s(7)=2.12,1.87,2.12$, and -0.18 , all $p$ s $>.05$, for $\mathrm{G} 24, \mathrm{G} 27, \mathrm{P} 64$, and S53, respectively].

Transfer performance was significantly better than chance $[t \mathrm{~s}(7)=7.76,4.07$, and 8.27 for $\mathrm{G} 24, \mathrm{G} 27$, and S53, respectively, $p s<.01$, and $t(7)=2.96$ for $\mathrm{P} 64$, $p<.05]$.

The starlings did notice that the patterns were different from baseline patterns, however. On the first session of transfer, the starlings responded before the end of the listening period on $30 \%$ of the trials. The birds very rarely responded too early with the baseline patterns. These early responses for the transfer patterns were undoubtedly a result of the offset of sound presentation serving as a cue for the bird to respond. The transfer patterns had long pauses in them, and the birds must have perceived these pauses as signaling the beginning of the response period.

\section{Discussion}

Removing the $\mathrm{O}$ element from the patterns and replacing them with silence did not affect the starlings' identification of the patterns. This is consistent with the hypothesis

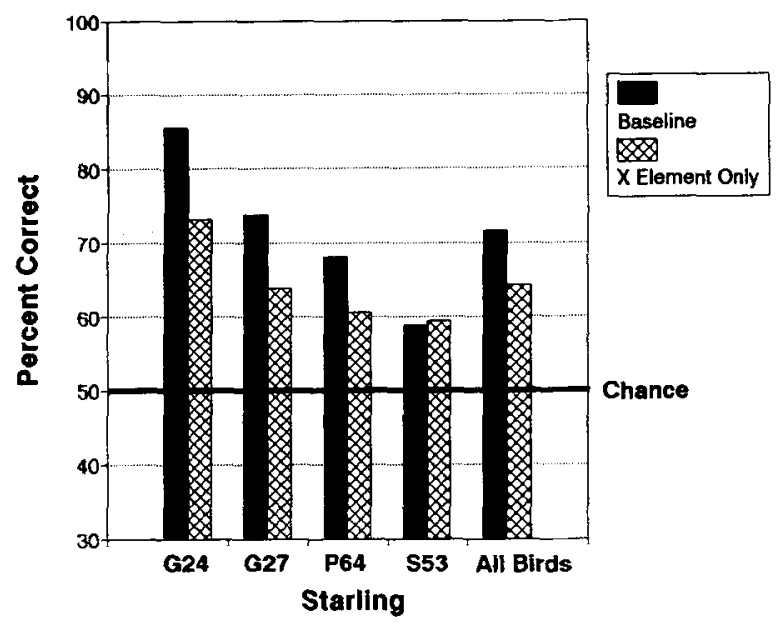

Figure 6. Baseline and transfer discrimination performances for the starlings in Experiment 6. Baseline data are the mean baseline performances on the eight blocks of 20 trials preceding the transfer test. Transfer data are the mean transfer performances on the eight blocks of 20 trials with the $O$ element replaced by a 100 -msec silent interval. that the starlings perceived the patterns as separate groups of $\mathrm{X}$ and $\mathrm{O}$ elements. The identical elements may have been grouped together to form perceptual streams (Bregman, 1990).

It is not clear from these results whether attention could be shifted between the two streams. If the starlings could perceive only the $X$ stream, but not the $O$ stream, they would be unable to identify patterns with the $X$ elements replaced by silent intervals. Alternatively, the starlings might have been able to identify the patterns by attending to either the $\mathrm{X}$ or the $\mathrm{O}$ element. This question was addressed in the next experiment.

\section{EXPERIMENT 7 Auditory Streaming: $O$ Element as Figure}

In this experiment, the $\mathrm{X}$ elements were replaced with 100 -msec silent intervals. If the starlings could identify the patterns by the rhythmic stream of $\mathrm{O}$ elements, this change in the patterns would not cause much of a decrement in performance. However, if the starlings attended only to the $X$ element in the baseline discrimination, discrimination performance would be disrupted.

\section{Method}

Subjects. The same 4 starlings served as subjects.

Apparatus. The apparatus is described in Experiment 1.

Stimuli. The $O$ element remained the same as in the baseline patterns. The $\mathrm{X}$ element was replaced with a 100 -msec silent interval. Thus, Pattern $A$ in this transfer was --.--OOO, where the dash represents a silent interval, and Pattern B was O-O-OO--.

Procedure. Each starling received i60 trials of the transfer patterns, as described in the procedure for the transfer tests in Experiment 1.

\section{Results}

The results for the 4 starlings are shown in Figure 7. Replacing the $\mathrm{X}$ element with silent intervals did not affect the ability to identify the patterns. Average discrimination performance was $66.9 \%$ on the baseline patterns and $70.5 \%$ on the transfer patterns $[t(7)=-1.06, p>$ $.05]$. This result was consistent for the 4 birds; there were no significant differences between performance in the transfer condition and baseline $[t \mathrm{~s}(7)=-0.56,-2.22$, 0.91 , and -1.18 for Starlings G24, G27, P64, and S53, respectively, all $p s>.05]$.

All of the starlings performed better than chance with the transfer patterns $[t \mathrm{~s}(7)=9.96,6.32$, and 4.51 for $\mathrm{G} 24, \mathrm{G} 27$, and S53, $p \mathrm{~s}<.01$, and $t(7)=3.21$ for $\mathrm{P} 64$, $p<.05]$.

\section{Discussion}

The performance of the starlings was unaffected by when the $\mathrm{X}$ elements were replaced by silent intervals. Thus, the starlings were able to identify the patterns at greater than chance levels when either of the elements was removed. This implies that the starling's attention could shift to either of the two rhythmic organizations of the patterns. Either pattern could be perceived as a figure against the background of the other. 


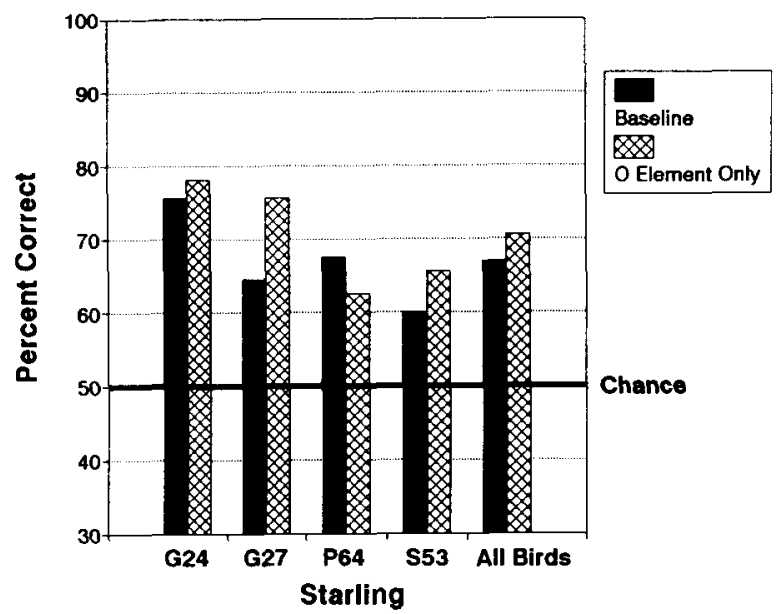

Figure 7. Baseline and transfer discrimination performances for the starlings in Experiment 7. Baseline data are the mean baseline performances on the eight blocks of 20 trials preceding the transfer test. Transfer data are the mean transfer performances on the eight blocks of 20 trials with the $X$ element replaced by a 100 -msec silent interval.

The finding that either of the elements could be removed without disrupting performance is remarkable. Any theory of stimulus generalization would predict that distorting the stimulus as greatly as was done in Experiments 6 and 7 should greatly affect discrimination. That discrimination was not significantly affected strongly indicates that the repeating patterns were organized perceptually by grouping identical stimulus elements.

The data from Experiments 6 and 7 point to two possible explanations for the starlings' performance. One is that the starlings perceived the elements as splitting into two distinct perceptual streams. The starlings could attend to either of the two streams, and this ability would account for the lack of performance decrement on the transfer tests in Experiments 6 and 7.

A second explanation, however, can account for the data equally well. The starlings may have been discriminating on the basis of the run length of identical elements (Albert Bregman, personal communication, May 22, 1992). In the baseline patterns, the longest run in Pattern A was five elements, and the longest run in Pattern B was two elements. In Experiment 6, the longest run lengths did not change in the transfer patterns, so good performance would be expected. In the transfer patterns of Experiment 7, the longest run length of Pattern $A$ was three, and the longest run of Pattern B was two. Thus Pattern A maintained the longest run from the baseline patterns to the transfer patterns. As would be expected from the longest run hypothesis, there was no decrement in performance.

Both the streaming hypothesis and the run-length hypothesis can account for the data in all the experiments. It is important to point out that both hypotheses assume that the starling groups identical elements perceptually. The question is how the starlings make use of the grouped elements. We will return to this question below.

\section{GENERAL DISCUSSION}

The results from these experiments with starlings provide some answers to the question of what a songbird perceives in a series of acoustic elements.

\section{Perception of Pattern Structure}

There is evidence that the starlings did select from among the possible organizations of the acoustic elements. In Experiment 2, the starlings discriminated the patterns with the novel starting points as easily as they did the baseline training starting points. Furthermore, the reorganization of the elements in Experiment 3 severely disrupted the ability to identify the patterns. The transfer in this experiment actually caused 1 of the starlings to reverse its responses to the two patterns in a way that was understandable from the organization of the patterns. Two of the starlings were not affected at all by the introduction of novel elements in Experiment 4, and 1 of the starlings was still able to identify the patterns even when the positions of the elements were reversed in Experiment 5. The results of Experiments 1-5 indicate that for starlings, perception is an active process in which a perceptual organization is selected from among a number of alternatives.

\section{Perception of Pattern Elements}

The starlings did perceive and remember features of the two elements that formed the patterns. That is, the perception of these patterns was not entirely abstract. The first evidence of this was found in acquisition. The discrimination between the repeating patterns was extremely difficult for 2 of the starlings. The experiment was designed to make it impossible to discriminate between the patterns on the basis of the features of individual elements. Both patterns were constructed from the same two elements, and both could begin at any of several starting points. Any features of the elements were irrelevant to the task. The slow acquisition of the patterns is indirect evidence that, in spite of the design of the experiment, 2 of the starlings initially attempted to solve the discrimination on the basis of features of the elements. Supporting evidence comes from studies with pitch patterns, in which starlings have been shown to discriminate more readily on the basis of pattern elements than of pattern structure (Braaten et al., 1990; Hulse \& Cynx, 1985, 1986; Hulse et al., 1984; Hulse et al., 1990b; Page et al., 1989).

More direct evidence that the starlings perceived and encoded features of the pattern elements comes from the results of Experiments 4 and 5. In those experiments, some of the starlings' performance was affected adversely by altering the pattern elements. This was especially true for the starlings that had difficulty learning the discrimination, and it was especially true when the positions of the elements in the patterns were reversed. If the starlings' representation of the patterns had been entirely abstract, these alterations would not have affected their discrimination performance. 


\section{Perception of Auditory Streams}

The results of Experiments 6 and 7 indicate that the starlings may have perceived these patterns as perceptual streams. Removing either of the two elements and replacing them with silent intervals did not eliminate discrimination. The starlings may have attended to the figural stream formed by either of the two elements, while the other element was perceived as background. If so, this would be the first demonstration of auditory streaming by a nonhuman animal.

However, the hypothesis that the starlings were discriminating on the basis of the run length of identical elements accounts for the data equally well. The present studies leave this question unresolved. Future research could address this problem in two ways. First, starlings could be trained with patterns that contain identical run lengths of elements, with the order of the runs rearranged between the patterns. Better still, starlings could be trained to discriminate patterns with more than two elements. For example, Bregman and Campbell (1971) presented humans with patterns consisting of three different high-pitched tones and three different low-pitched tones. The highpitched tones were perceived as forming one stream, and the low-pitched tones formed another.

The study of stream segregation in animals remains a potentially fruitful area for investigation. The features that cause elements to form separate streams have been a subject of great interest in human auditory perception (Bregman, 1990). Perhaps other relationships among the elements, such as frequency similarity or proximity, could be used by starlings to group the elements into streams.

The functional importance of auditory streaming is that it allows an animal to attend selectively to a sequence in the face of background noise. For a songbird, streaming could enable the bird to perceive a particular song against the raucous background of other sounds. The streaming of elements in more natural settings is also an important area for future investigation.

Of course, the grouping of acoustic elements could also play a role in parsing meaningful units of perception within a song. A good deal is known about the stimulus dimensions that are utilized by songbirds in making song discriminations (see, e.g., Brooks \& Falls, 1975; Emlen, 1972; Nelson, 1988), but little is known about functionally important groupings of elements within song (but see Cynx, 1990). Studies such as those reported here, but with the use of continuously repeating natural song syllables rather than artificial acoustic elements, could yield important information relevant to this question.

\section{REFERENCES}

Attneave, F., \& Olson, R. K. (1971). Pitch as a medium: A new approach to psychophysical scaling. American Journal of Psychology, 84, 147-166.

BRAATEN, R. F. (1991). Auditory perceptual organization by a songbird, the European starling (Sturnus vulgaris). Unpublished doctoral dissertation, The Johns Hopkins University, Baltimore.
Braaten, R. F., \& Hulse, S. H. (1991). A songbird, the European starling (Sturnus vulgaris), shows perceptual constancy for acoustic spectral structure. Journal of Comparative Psychology, 105, 222-231.

Braaten, R. F., Hulse, S. H., \& Page, S. C. (1990). Discrimination and classification of rising and nonrising pitch patterns by the European starling (Sturnus vulgaris). Animal Learning \& Behavior, 18, 352-364.

Bregman, A. S. (1990). Auditory scene analysis: The perceptual organization of sound. Cambridge, MA: MIT Press.

Bregman, A. S., Abramson, J., Doehring, P., \& Darwin, C. J. (1985). Spectral integration based on common amplitude modulation. Perception \& Psychophysics, 37, 483-493.

Bregman, A. S., \& Campiell, J. (1971). Primary auditory stream segregation and perception of order in rapid sequences of tones. Journal of Experimental Psychology, 89, 244-249.

BrooKs, R. J., \& FALLS, J. B. (1975). Individual recognition by song in white-throated sparrows: III. Song features used in individual recognition. Canadian Journal of Zoology, 53, 1749-1761

Chalikia, M. H., \& Bregman, A. S. (1989). The perceptual segregation of simultaneous auditory signals: Pulse train segregation and vowel segregation. Perception \& Psychophysics, 46, 487-496.

CYNX, J. (1990). Experimental determination of a unit of song production in the Zebra finch (Taeniopygia guttata). Journal of Comparative Psychology, 104, 3-10.

D'AмAтo, M. R. (1988). A search for tonal pattern perception in cebus monkeys: Why monkeys can't hum a tune. Music Perception, 5, 452-480.

D'Amato, M. R., \& Salmon, D. P. (1982). Tune discrimination in monkeys (Cebus apella) and in rats. Animal Learning \& Behavior, 10, 126-134.

D'Amato, M. R., \& Salmon, D. P. (1984). Processing of complex auditory stimuli (tunes) by rats and monkeys (Cebus apella). Animal Learning \& Behavior, 12, 184-194.

Dannenbring, G. L., \& Bregman, A. S. (1978). Streaming vs. fusion of sinusoidal components of complex tones. Perception \& Psychophysics, 24, 369-376.

DeUtsch, D. (Ed.) (1982). The psychology of music. New York: Academic Press.

Divenyi, P. L., \& HiRsh, I. J. (1978). Some figural properties of auditory patterns. Journal of the Acoustical Society of America, 64, 1369-1385.

Dooling, R. J., Brown, S. D., Park, T. J., Okanoya, K., \& Soli, S. D. (1987). Perceptual organization of acoustic stimuli by budgerigars (Melopsittacus undulatus): I. Pure tones. Journal of Comparative Psychology, 101, 139-149.

Dowling, W. J., \& HaRwood, D. L. (1986). Music cognition. Orlando, FL: Academic Press.

EMLEN, S. T. (1972). An experimental analysis of the parameters of bird song eliciting species recognition. Behaviour, 41, 130-171.

GARNER, W. R. (1974). The processing of information and structure. Potomac, MD: Erlbaum.

GARNER, W. R., \& GoTTWALD, R. L. (1968). The perception and learning of temporal patterns. Quarterly Journal of Experimental Psychology, 20, 97-109.

GreENE, S. L. (1984). Feature memorization in pigeon concept formation. In M. L. Commons, R. J. Herrnstein, \& A. R. Wagner, (Eds.), Quantitative analyses of behavior, discrimination processes (pp. 209-232). Cambridge, MA: Ballinger.

HaNDEL, S. (1974). Perceiving melodic and rhythmic auditory patterns Journal of Experimental Psychology, 103, 922-933.

HANDEL, S., \& BUFFARDI, L. (1968). Pattern perception: Integrating information presented in two modalities. Science, 162, 1026-1028.

HANDEL, S., \& LEWIS, W. E. (1970). Effect of practice on the perception of temporal patterns. Quarterly Journal of Experimental Psychology, 22, 97-108.

HANDEL, S., \& TODD, P. (1981). Segmentation of sequential patterns. Journal of Experimental Psychology: Human Perception \& Performance, $7,41-55$

Hulse, S. H., \& CYNX, J. (1985). Relative pitch perception is constrained by absolute pitch in songbirds (Mimus, Molothrus, and Sturnus). Journal of Comparative Psychology, 99, 176-196. 
Hulse, S. H., \& CYNX, J. (1986). Interval and contour in serial pitch perception by a passerine bird, the European starling (Sturnus vulgaris). Journal of Comparative Psychology, 100, 215-228.

Hulse, S. H., CynX, J., \& HuMPal, J. (1984). Absolute and relative pitch perception in serial pitch perception by birds. Journal of Experimental Psychology: General, 113, 38-54.

Hulse, S. H., Page, S. C., \& BraAten, R. F. (1990a). An integrative approach to auditory pattern perception by songbirds. In M. Berkley \& W. Stebbins (Eds.), Comparative perception: Vol. 2. Complex signals (pp. 3-34). New York: Wiley.

Hulse, S. H., Page, S. C., \& Braaten, R. F. (1990b). Frequency range size and the frequency range constraint in auditory perception by European starlings (Sturnus vulgaris). Animal Learning \& Behavior, $18,238-245$.

MCADams, S. (1989). Segregation of concurrent sounds: I. Effects of frequency modulation coherence. Journal of the Acoustical Society of America, 86, 2148-2159.

NELSON, D. A. (1988). Feature weighting in species song recognition by the field sparrow (Spizella pusilla). Behaviour, 106, 158-182.

Page, S. C., Hulse, S. H., \& CYNX, J. (1989). Relative pitch perception in the European starling (Sturnus vulgaris): Further evidence for an elusive phenomenon. Journal of Experimental Psychology: Animal Behavior Processes, 15, 137-146.
Preusser, D. (1972). The effect of structure and rate on the recognition and description of auditory temporal patterns. Perception \& Psychophysics, 11, 233-240.

Preusser, D., Garner, W. R., \& Gottwald, R. L. (1970). Perceptual organization of two-element temporal patterns as a function of their component one-element patterns. American Journal of Psychology, 83, 151-170.

ROYER, F. L. (1967). Sequential complexity and motor response rates. Journal of Experimental Psychology, 74, 199-202.

ROYER, F. L., \& GARNER, W. R. (1966). Response uncertainty and perceptual difficulty of auditory temporal patterns. Perception \& Psychophysics, 1, 41-47.

Royer, F. L., \& Garner, W. R. (1970). Perceptual organization of nine-element auditory temporal patterns. Perception \& Psychophysics, $7,115-120$

Warren, R. M., Obusek, C. J., Farmer, R. M., \& Warren, R. P. (1969). Auditory sequence: Confusion of patterns other than speech or music. Science, 164, 586-587.

(Manuscript received April 27, 1992; revision accepted for publication March 26, 1993.) 